\title{
Protein (A-FABP) in Obese Patients before and after Omega Loop Gastric Bypass: 1 Year Follow up
}

\author{
Mohamed I Hassan, Ahmed Saeed Saad, Mohammed Attia Elsayed \\ Department of General Surgery, Faculty of Medicine, Ain Shams University, Egypt
}

Introduction: A-FABP (Adipocyte fatty acid-binding protein) has been shown to affect lipid metabolism, insulin sensitivity, and lipolysis furthermore, studies also found that A-FABP is a key mediator for obesity-related cardiovascular disease and is usually associated with metabolic syndrome. Although A-FABP is thought to be a cytoplasmic protein, it is also detectable in the human bloodstream. Recent evidence showed that circulating A-FABP level to be an independent predictor of the development of metabolic syndrome and diabetes.

Objective: The association of A-FABP with obesity and the decrease of its level after laparoscopic omega loop gastric bypass as indicator of improvement of metabolic syndrome.

Patients and methods: Sixty-five morbid-obese patients had laparoscopic omega loop gastric bypass at Ain Shams University Hospitals and cure hospital from February 2018 to June 2019 with a mean age of $42.5 \pm 9.6$ years were included in the study with comparative to non-obese control group. The A-FABP was measured before Laparoscopic omega loop gastric bypass and after 1 year of follow-up.

Results: FABP1 levels in the obese $10.5(8.25-14.25) \mathrm{P}<0.01$, as compared to the control group 1.5 $(0.675-2.625)$ analysis revealed that the FABP1 levels were positively correlated with BMI $(P<0.01)$. After weight loss, A-FABP plasmatic levels decreased significantly $[3.4(0.2-6)]$ ) $\mathrm{P}<0.01$.

With correlation to improvement of the laboratory investigations of the metabolic syndrome fasting, 2 hours postprandial blood glucose level (2hPP) and glycated hemoglobin (HbA1) cholesterol, triglycerides (TG), high-density lipoprotein cholesterol (HDL-C), low-density lipoprotein cholesterol (LDL-C)\} fasting serum insulin level. Highly sensitive C-reactive protein (hsCRP). Were also analyzed and they showed a significant difference between the obese patients and 1 year after surgery.

Conclusion: Serum A -FABP1 levels are markedly associated with obesity and correlated with an increase in BMI, and it markedly decreased with weight loss after omega loop gastric bypass.

Key words: A-FABP1, laparoscopic omega loop gastric bypass, metabolic, obesity.

\section{Introduction}

Obesity is identified to be caused by excessive accumulation of long-chain fatty acids (LCFA), especially triglycerides, in adipose and other tissues. $^{1}$

Morbid obesity is an extreme metabolic situation in which the imbalance of adipocytokines is amplified and presents a different regulatory pattern than that detected in non-morbid-obese patients. ${ }^{2}$

Adipocyte fatty acid-binding (A-FABP) is one of the known proteins in the mature adipocytes, it's known for its ability to bind fatty acids and related compounds throughout different cellular compartments, including the mitochondria, endoplasmic reticulum, peroxisomes, lipid droplets, and nucleus. ${ }^{3}$

A-FABP is composed of 127 amino acids and has a molecular weight of approximately $14-15 \mathrm{kDa}$, which is not only expressed in the cytoplasm but also the nucleus, and it can regulate the expression of some important genes of lipid metabolism. ${ }^{4}$

A-FABP has been shown to affect lipid metabolism, insulin sensitivity, and lipolysis furthermore, studies also found that A-FABP is a key mediator for obesity-related cardiovascular disease and is usually associated with metabolic syndrome. ${ }^{5}$

Although A-FABP is thought to be a cytoplasmic protein, it is also detectable in the human bloodstream. Recent evidence showed that circulating A-FABP level to be an independent predictor of the development of metabolic syndrome and diabetes. ${ }^{6}$

Most of the metabolic alterations markedly improved after weight reduction. We assume that 
A-FABP should be reduced with weight reduction in morbid-obese patients in correlation with inflammatory markers and after improving insulin sensitivity bariatric surgery has a significant effect on the management of morbidly obese patients with marked weight reduction and metabolic improvement in obesity-related comorbidities, such as type 2 diabetes mellitus (T2DM), and hypertension and hyperlipidemia., ${ }^{78}$

Omega loop gastric bypass a is rapidly evolving bariatric procedure offering marked weight loss and a decrease in the incidence of obesity-related comorbidities in morbidly obese patients. ${ }^{8}$

Aim: In the current study, we aimed to investigate the correlation of FABP1 with obesity and the effect of laparoscopic omega loop gastric bypass on its level after 1 year of surgery.

\section{Patients and methods}

Sixty-five morbid-obese patients who had laparoscopic omega loop gastric bypass at Ain shams University Hospitals and Cure Hospital from February 2018 to June 2019 with a mean age of $42.5 \pm 9.6$ years were included in the study and. The patient group was compared to a non-obese control group as regard (A-FABP) blood levels were compared pre-operatively and 1-year post operatively. The correlation between the decrease of A FABP level with improvement of metabolic syndrome including diabetes was also noted.

Informed consent was obtained; the procedure and the possible complications were explained to all the patients.

\section{Anthropometric and biochemical measurements}

Full medical history and clinical examination and blood pressure were measured. Anthropometric measurements (weight, height, BMI calculation, and waist circumference).

\section{Laboratory investigations}

Fasting, 2 hours post-prandial blood glucose level $(2 \mathrm{hPP})$ and glycated hemoglobin (HbA1c)

Lipid profile \{cholesterol, triglycerides (TG), highdensity lipoprotein cholesterol (HDL-C), low-density lipoprotein cholesterol (LDL-C)\}, fasting serum insulin level.

Highly sensitive C- reactive protein (hSCRP).

Insulin resistance was evaluated using a homeostasis model assessment of insulin resistance (HOMA-IR) as follows: HOMA-IR = fasting plasma glucose $(\mathrm{mg} /$ $\mathrm{dL}) \times$ fasting serum insulin $(\mu \mathrm{U} / \mathrm{mL}) / 405$.
The A-FABP was measured by ELISA kit supplied by Elabscience Biotechnology Inc., USA* applied for in vitro quantitative determination of Human A-FABP concentrations in serum, plasma, and other biological fluids.

Venous blood was obtained from all patients in the morning after a 12-h overnight fast; the samples were then centrifuged, and the supernatant was used for laboratory testing. The serum FABP1 levels were measured by enzyme-linked immunosorbent assay (ELISA, Abcam, ab218261). The normal reference level of FABP1 according to the ELISA kit used was $6.36-19.0 \mathrm{ng} / \mathrm{ml}$. The sampling was done before Laparoscopic omega loop gastric bypass and after 1 year of follow-up.

\section{Surgical technique}

As shown Figure 1, along and narrow tubelike gastric pouch was created by applying one horizontal articulator Endo-GIA $®$ stapler (Covidien, Cincinnati, OH, USA) at the lesser curvature, crow's foot, and then four to six vertical $60-\mathrm{mm} \AA$ stapler cartridges were placed upwards toward the angle of His along a 36-Fr bougie. The jejunum $200 \mathrm{~cm}$ from the ligament of Treitz, was anastomosed endto-side tube, using a posterior 45-mm Endo-GIA $®$ stapler. The stapler defect was sutured anteriorly. The anastomosis was checked for leak by using both methylene-blue test and air bubble test.

Tube drain was inserted for 24 hours, we recommend early ambulation, and anticoagulant was given for 3 weeks

\section{Statistical methods}

Statistical analysis was performed by IBM SPSS statistics, version 25.0, IBM Corporation, USA, 2017-2018.

\section{Results}

The clinical and the metabolic, changes were markedly significant and analytical for all subjects before and 1 year after gastric bypass, including total cholesterol, triglycerides, waist-hip ratio, FFA, and insulin resistance assessed by HOMA-IR (all variables at $P<0.01$ ).

The weight was 100 (100-140) preoperatively and it was decreased 11 year after surgery 83 (68.7590.25 ) with marked improvement of BMI ratio from $40(29-40)$ to $28.25(26.5-32)$ post operatively, the change was $P<0.01$ Highly Significant (HS).

High level of A-FABP was noticed in obese patient 10.5 (8.25-14.25) when compared to the control group 1.5 (0.675-2.625) and it was markedly decreased 1 year after surgery with high significant changes $\mathrm{P}<0.01$. 
The decrease of A-FABP level was correlated with improvment of different parameters of metabolic syndrome as the decrease of $\mathrm{HbA1C}$ from 7.35 (6.357-8.6) to $6.3(5.6-6.9)$ and hsCRP that was markedly decreased from $0.6185(0.282-1.218)$

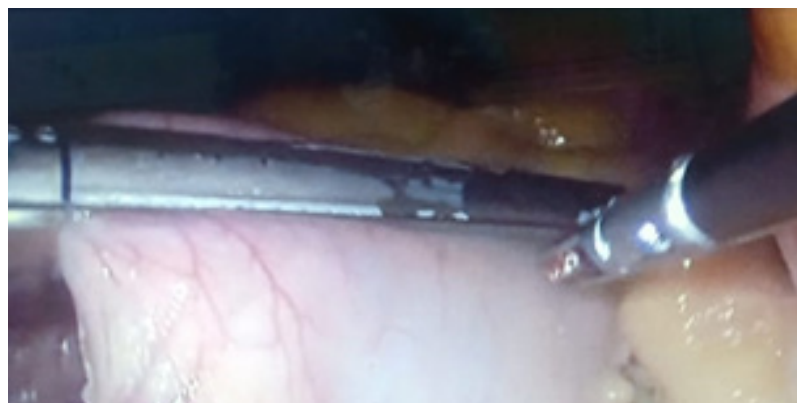

A

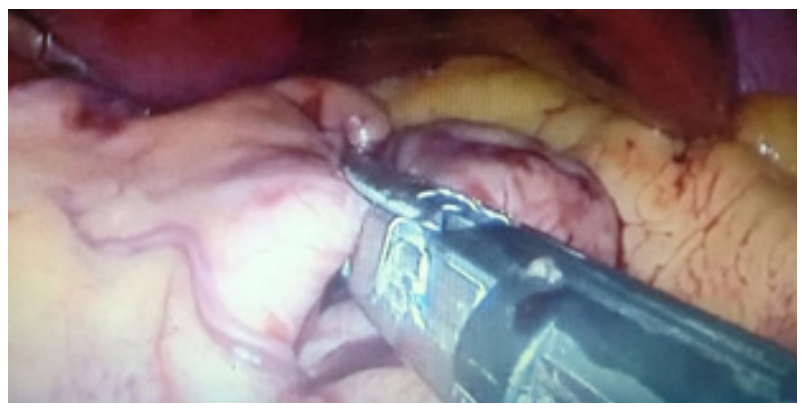

B to $0.4485(0.153-0.578)$ high significant changes $\mathrm{P}<0.01$. Significant improvement of $\mathrm{HDL}$ and its increase from $44.5(39.75-49.75)$ to 50.5 (43.2557.25) with significant change $P<0.05$.

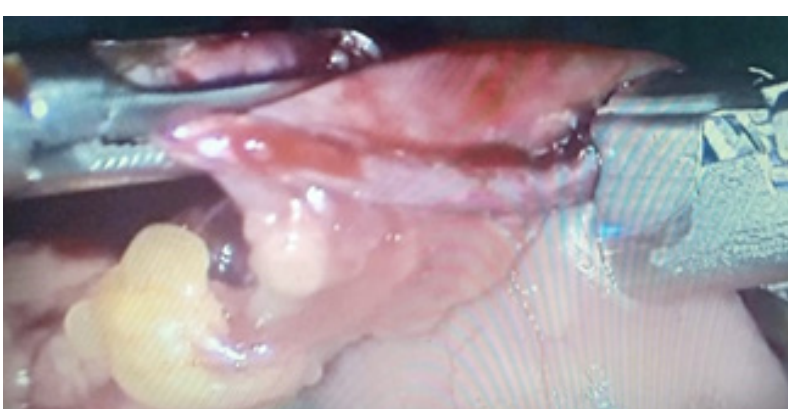

C

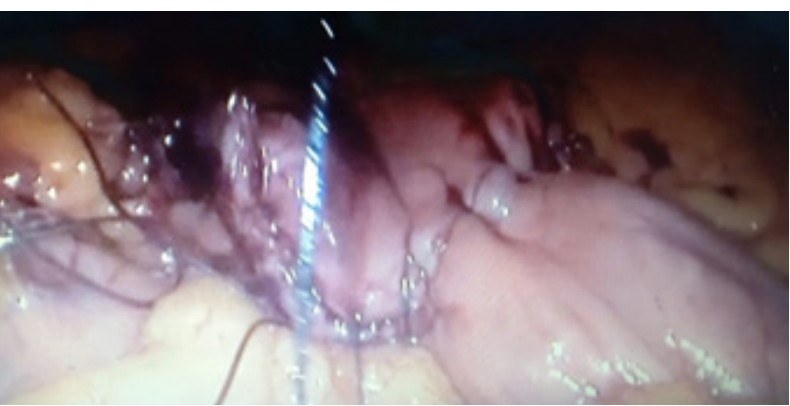

Fig 1a,b,c,d: Showed steps of omega loop gastric bypass.

Table 1: Statistical characteristics of different studied parameters among all the patients' before and after 1 year in comparable to the control group using Kruskall-Wallis test

\begin{tabular}{|c|c|c|c|c|c|}
\hline Parameter & Lyear after surgery & Pre Operative & Control group & $\mathbf{H}$ & $\mathbf{P}$ \\
\hline Weight (Kg) & $83(68.75-90.25)$ & $100(100-140)$ & $72.5(68-85.25)$ & 23.543 & $<0.01$ \\
\hline Height (cm) & $166.5(160-170.25)$ & $165(160-172.5)$ & $170(162.25-175)$ & 2.763 & $>0.05$ \\
\hline BMI $\left(\mathrm{Kg} / \mathrm{m}^{2}\right)$ & $28.25(26.5-32)$ & $40(29-40)$ & $26(24-28.25)$ & 35.238 & $<0.01$ \\
\hline Waist (cm) & $86(78-95.75)$ & $109(94.5-120.5)$ & $83.5(78.75-91.25)$ & 27.429 & $<0.01$ \\
\hline FBS (mg/dL) & $137.5(101.75-173.75)$ & $125.5(101.5-158.25)$ & $80(75.735-86)$ & 52.657 & $<0.01$ \\
\hline $2 \mathrm{hPP}(\mathrm{mg} / \mathrm{dL})$ & $191.4(147.25-262.75)$ & $204.5(170.75-275)$ & $97(89.74-104)$ & 51.223 & $<0.01$ \\
\hline HbA1c (\%) & $6.3(5.6-6.9)$ & $7.35(6.357-8.6)$ & $5.2(5-5.3)$ & 56.846 & $<0.01$ \\
\hline T.Chol (mg/dL) & $170.5(144.25-206.26)$ & $192(164-239.25)$ & $165(139-192)$ & 5.937 & $>0.05$ \\
\hline $\mathrm{TG}(\mathrm{mg} / \mathrm{dL})$ & $122.5(87.5-148)$ & $147(111.5-196.5)$ & $93(84.5-122.26)$ & 19.683 & $<0.01$ \\
\hline HDL-C (mg/dL) & $50.5(43.25-57.25)$ & $44.5(39.75-49.75)$ & $54(46.5-60.25)$ & 9.035 & $<0.05$ \\
\hline LDL-C (mg/dL) & $100.5(77.5-117.74)$ & $111.4(86.25-150.75)$ & $111(97-123.77)$ & 3.052 & $>0.05$ \\
\hline F.Insulin $(\mu \mathrm{IU} / \mathrm{mL})$ & $11.5(5-20.4)$ & $19(10.75-25)$ & $8.5(5.375-12)$ & 22.785 & $<0.01$ \\
\hline HOMA-IR & $3.94(2.175-7.9)$ & $5.5(3-9.375)$ & $1.64(0.975-2.225)$ & 39.109 & $<0.01$ \\
\hline A-FABP (ng/mL) & $3.4(0.2-6)$ & $10.5(8.25-14.25)$ & $1.5(0.675-2.625)$ & 41.389 & $<0.01$ \\
\hline hsCRP (mg/dL) & $0.4485(0.153-0.578)$ & $0.6185{ }_{1.218)^{(0.282-}}$ & $0.155(0.079-0.235)$ & 2.6106 & $<0.01$ \\
\hline
\end{tabular}

Q1: $25^{\text {th }}$ percentile; Q3: $75^{\text {th }}$ percentile. $\quad \mathrm{P}<0.01$ Highly Significant $(H S) . \quad P<0.05$ Significant(S). $P>0.05$ Non Significant. 


\section{Discussion}

A-FABP is a member of the FABP family which is markedly expressed in adipose tissue employing adipocytes.

FABP1 is more commonly used as a biomarker related to kidney, liver diseases and type 1 diabetes (T1DM). ${ }^{9}$

It is a fatty acid transporter, however its role in the different clinical changes that are associated with obesity is still unclear, our research on the role of FABP1 in obesity and its level after weight reduction is one of few types of research that follow that issue as many types of research based on the cell and animal models only. ${ }^{10}$

Many studies had shown the marked association between obesity and various diseases, mainly type 2 diabetes mellitus..$^{11}$ In addition to lipid accumulation and free fatty acids (FFA) release, adipocytes and other adipose tissue release signaling proteins known as adipokines. A-FABP was recently discussed as a new adipokine that could be linked to obesity and impaired glucose metabolism. ${ }^{11}$

Our study focuses mainly on 2 aspects, first is the expression of FABP1 and its relation to the BMI, second is the different changes in the serum FABP1 levels 1 year after laparoscopic omega loop gastric bypass.

Binas found that the FABP1 knockout mice showed marked reduced utilization of fatty acids, decreased insulin resistance which suggested that FABP1 I marked factor in the intracellular utilization of fatty acids and energy metabolism. ${ }^{13}$

Other studies indicated that the overexpression of FABP1 in cells can markedly increase the rate of fatty acids uptake, which could be explained that FABP1 can increase the dissociation of LCFAs from the cell membrane and increase the water solubility of the LCFAs and membrane partition coefficient, thus increasing the rate at which the LCFAs are transported to the membrane receptor. ${ }^{14}$

FABP1 was also found to promote the vascular endothelium growth by increasing the levels of fatty acids in the blood, thereby promoting the expression of vascular endothelial growth factors and promoting the development of hepatic cancer. ${ }^{15}$

In our study, we showed the high serum level of $A$ FABP in the obese patients and the marked reduction Of its level after one 1 year of omega loop gastric bypass and we showed the marked association of the serum level of A- FABP with the anthropometric measurement and lipid profile.
Our findings are consistent with those of $\mathrm{HU}$ YOU et $\mathrm{a}^{16}$ Serum FABP1 levels are markedly correlated with obesity, and he found that with the decrease in BMI after laparoscopic sleeve gastrectomy, FABP1 levels also markedly decreased postoperatively.

However, our study was not consistent with those of Julia Engle et $\mathrm{al}^{17}$ who had 2 studies one of them had showed a steady level of AFABP hadn't changed 1 year after weight loss, and the second study showed a significant increase in its level 3 months after weight loss.

She assumed that A-FABP is not only a biomarker of obesity and metabolic syndrome but also it a marker of weight changes in dynamic situations.

We hope that our findings may encourage researchers and clinicians working on obesity to adopt more research as that FABP1 may become an important indicator of obesity.

\section{Conclusion}

Serum A -FABP1 levels are markedly associated with obesity and correlated with an increase in BMI, and it markedly decreased with weight loss after omega loop gastric bypass.

\section{References}

1. Wang G, Bonkovsky $\mathrm{HL}$, de Lemos $\mathrm{A}$, et al: Recent insights into the biological functions of liver fatty acid-binding protein 1. J Lipid Res. 2015; 56(12): 2238-47.

2. Vendrell J, Broch M, Vilarrasa N, et al: Resistin, adiponectin, ghrelin, leptin and pro-inflammatory cytokines: Relationships in obesity. Obes Res. 2004; 12: 962-971.

3. Furuhashi M, Saitoh S, Shimamoto K, et al: AcidBinding protein 4 (FABP4): Pathophysiological insights and potent clinical biomarker of metabolic and cardiovascular diseases. Clinical Medicine Insights Cardiology. 2014; 8(3): 23-33.

4. Prinetti A, Mitro N: FABP1 in wonderland. $\boldsymbol{J}$ Neurochem. 2016; 138(3) :371-3.

5. Xu A, Vanhoutte PM: Adiponectin and adipocyte fatty acid-binding protein in the pathogenesis of the cardiovascular disease. American Journal of Physiology-Heart and Circulatory Physiology. 2012; 302(6): 1231-1240.

6. Tso AW, Xu A, Sham PC, et al: Serum adipocyte fatty acid-binding protein as a new biomarker predicting the development of type 2 diabetes: A 10-year prospective study in a Chinese cohort. Diabetes Care. 2007; 30(10): 2667-72. 
7. McIntosh $A L$, Huang $H$, Storey $S M$, et al: Human FABP1 T94A variant impacts fatty acid metabolism and PPAR-alpha activation in cultured human female hepatocytes. $\mathbf{A m} \boldsymbol{J}$ Physiol Gastrointest Liver Physiol. 2014; 307(2): G164-G176.

8. Chevallier JM, Arman GA, Guenzi M, et al: One thousand single anastomosis (Omega Loop) gastric bypasses to treat morbid obesity in 7 years: Outcomes show few complications and good efficacy. Obes Surg. 2015; 25: 951-958.

9. Novellas $C J$, Speiser JL, Tremblay $M$, et al: Elevated FABP1 se [1] rum levels are associated with poorer survival in acetaminophen [1] induced acute liver failure. Hepatology. 2017; 65(3): 938-49.

10. Milligan S, Martin GG, Landrock $D$, et al: Ablating both FABP1 and Scp2/Scpx (TKO) induces hepatic phospholipid and cholesterol accumulation in high fat-fed mice. Biochim Biophys Acta Mol Cell Biol Lipids. 2018; 1863(3): 323-338.

11. Haslam DW, James WP (2005): Obesity. Lancet. 2005; 366(9492): 1197-1209.

12. Gustafson B: Adipose tissue, inflammation and atherosclerosis. J Atheroscler Thromb. 2010; 30;17(4): 332-41.

13. Binas B, Erol E: FABPs as determinants of myocellular and hepatic fuel metabolism. Mol Cell Biochem. 2007; 299(1): 75-84.

14. Dharmarajan S, Newberry EP, Montenegro G, et al: Liver fatty acid-binding protein (L-Fabp) modifies intestinal fatty acid compo [1] sition and adenoma formation in ApcMin/+ mice. Cancer Prev Res. 2013; 6(10): 1026-37.

15. Van Nieuwenhoven FA, Verstijnen CP, Van Eys GJ, et al: Fatty acid transfer across the myocardial capillary wall: No evidence of a sub [1] stantial role for cytoplasmic fatty acid-binding protein. $\boldsymbol{J}$ Mol Cell Cardiol. 1994; 26(12): 1635-47.

16. You, H, Wen, X, Zhu, C, et al: Serum FABP1 levels correlate positively with obesity in chinese patients after laparoscopic sleeve gastrectomy: A 12-month follow-up study. Obes Surg. 2020; 30: 931-940.

17. Engl J, Ciardi C, Tatarczyk T, et al: A-FABP -a bio [1] marker associated with the metabolic syndrome and/or an indicator of weight change? Obesity (Silver Spring). 2008; 16: 1838-42. 